\title{
EL DEBATE SOBRE EL CARÁCTER JURÍDICO DEL DERECHO DE GENTES EN HISPANOAMÉRICA DURANTE EL SIGLO XIX: LA CONTRAPOSICIÓN ENTRE JOSÉ SILVA SANTISTEBAN Y ANDRÉS BELLO
}

\author{
THE DEBATE ON THE LEGAL CHARACTER OF THE LAW OF NATIONS IN SPANISH \\ AMERICA DURING THE 19TH CENTURY: THE CONTRAST BETWEEN JOSÉ SILVA \\ SANTISTEBAN AND ANDRES BELLO
}

Pablo Rosales Zamora*

\section{Resumen}

La evolución del derecho de gentes en Hispanoamérica durante el siglo XIX ha sido poco estudiada. El presente trabajo tiene como objetivo afrontar ese vacío a partir del análisis de las posturas de José Silva Santisteban y Andrés Bello sobre la juridicidad del derecho internacional. Se sostendrá que el primero concibe al derecho internacional como Derecho, mientras que el segundo se aproxima a considerarlo, simplemente, como una "colección de leyes". Para entender este debate, se acudirá a los trabajos de Becker Lorca y Richard Morse, sustentando que Silva Santisteban se inscribe en una postura neotomista y Bello, en una aproximación utilitarista empirista del derecho internacional.

Palabras clave: Derecho de gentes - neotomismo - empirismo - juridicidad - Hispanoamérica.

\begin{abstract}
The evolution of law of nations in Spanish America during the $19^{\text {th }}$ century has been few studied. The present work has as aim covering that gap with the analysis on the José Silva Santisteban and Andres Bello's approaches on the legality of international law. This article will hold that the first author conceives properly international law as law, in contrast to the second one, who considers it only as a "collection of laws". For understanding this debate, the article will resort to the works of Becker Lorca and Richard Morse, holding Silva Santisteban

* Doctorando en Derecho y Ciencia Política por la Universidad Nacional Mayor de San Marcos (UNMSM). Profesor de Derecho Internacional en la Facultad de Derecho de la Universidad de San Martín de Porres (USMP) y de Derecho Internacional Privado en la Facultad de Derecho y Ciencia Política de la Universidad Católica Sedes Sapientiae (UCSS). Asesor en la Oficina de Derecho Internacional Público del Ministerio de Relaciones Exteriores del Perú.

El presente artículo está dedicado al profesor Silvio Mezarina García; asimismo, se escribe como tributo a la memoria del padre Armando Nieto Vélez S.J. (1931-2017).
\end{abstract}


represented a neo-thomist perspective and, by the contrary, Bello sustained a utilitarian and empiricist approach of international law.

Keywords: Law of nations - neothomism - empiricism - legality - Spanish America.

\section{Introducción}

Como es sostenido por la mayoría de historiadores del derecho internacional, a partir de los tratados de la Paz de Westfalia de 1648, se produjo la consolidación del Estado moderno y, con ello, la aparición de la disciplina del derecho internacional clásico (Cassese, 2013, pp. 31 y ss.; Franca, 2006, pp. 87 y ss.; Murphy, 2012, p. 22; Truyol y Serra, 1998, pp. 72-73). El llamado "derecho de gentes" ha sufrido una evolución desde entonces, cuya centralidad historiográfica ha girado en torno al llamado ius publicum europeum, prestándose poca atención a la producción bibliográfica sobre esta rama del Derecho en otros lugares del orbe (Armitage, D.; Pitts, J. 2017, pp. 1 y ss.). Especialmente, poco se ha escrito sobre los internacionalistas de la etapa postcolonial en América, durante el siglo XIX.

En su libro O espelho de Próspero: cultura e idéias nas Américas (1988), Morse afirma que América tiene una prehistoria europea que, si bien se caracterizó por una matriz cultural e intelectual común, esta base se bifurca, entre el siglo XII y el siglo XVII, en las llamadas opción ibérica y opción política inglesa, cuyas consecuencias en la mirada del derecho de gentes se pretenden investigar aquí, en plena efervescencia del siglo XIX (1988: pp. 26 - 72). En el caso ibérico, tenemos a un gran pensador que fue Francisco de Vitoria, el mayor representante de la Escuela de Salamanca, que opta en sus obras por un enfoque neotomista, en especial en la Relectio de Indis; en cambio, la vertiente inglesa se inclinó por un enfoque empirista, en el pensamiento de autores como Locke y Hobbes (Morse 1988: pp. 59-66). En esta tensión, la visión de Vitoria, que influirá en Hugo Grocio, supone el reconocimiento de un derecho de gentes desde una mirada iusnaturalista, que se inclina en aceptarlo como rama jurídica que no se puede desconocer (Gómez Robledo, 1989: pp. 30 y ss.), mientras que Hobbes niega su juridicidad. Una visión respalda el derecho de gentes y otra visión descarta, de plano, su esencia jurídica.

Esta discusión va a dar origen en Europa, por un lado, a una serie de negadores de la juridicidad del derecho internacional y, por otro lado, a defensores de esta. Siguiendo esta división, se ha identificado dos grandes autores sudamericanos del siglo XIX que encarnan la división teórica esgrimida por Morse. Por una parte, se encuentra el peruano Silva Santisteban, que se inclinó por defender el derecho internacional como ciencia "que trata de arreglar las relaciones internacionales según los principios eternos de justicia” (1856, p. 57). Por otra parte, Bello, estudioso venezolano que radicó en Chile, sostuvo que el derecho de gentes es, simplemente, una "colección de leyes o reglas generales de conducta que las naciones o Estados deben observar entre sí para su seguridad y bienestar común” (1864, p. 13).

A partir del modelo teórico elaborado por Morse, la presente investigación plantea, en este contexto, la interrogante de si es posible encontrar las representaciones de la Iberoamérica neotomista y la Angloamérica empirista en la literatura del derecho de gentes en pleno siglo XIX. En este esquema, se sostendrá que Silva Santisteban es representante de la primera corriente, al 
basarse este en el sostenimiento de la tesis de la existencia del derecho internacional y que Bello, al negar la existencia del derecho internacional, puede ser entendido como representante de la escuela empirista. Esta hipótesis se construirá a partir de las obras centrales de ambos autores que son, respectivamente, Curso de derecho internacional o de gentes y Principios del Derecho Internacional.

\section{Negación y afirmación del derecho de gentes}

Usualmente, se considera como fecha de inicio del derecho internacional al siglo XVII, con los tratados de la Paz de Westfalia ${ }^{1}$. Y esto se debe a que tales tratados representan un momento clave porque el Estado moderno se libera de dos factores de presión que le restaban protagonismo. Por un lado, y desde un punto de vista externo al Estado, el Imperio y la Iglesia Católica se ven desmejorados en su posición de poder con la entrada en vigor de tales tratados; por otro lado, y desde un punto de vista interno, el feudalismo se va desplazando ante la solidez estructural de la recaudación tributaria del Estado (Clapham 2012: pp. 2-7; Flórez 1999: pp. 119122).

Pero no siempre hubo una aceptación unánime de la esencia jurídica del derecho internacional. En ese contexto, uno de los puntos preliminares a resolver en el presente trabajo para abordar las posturas de Silva Santisteban y Bello es determinar el quid del debate relativo a la negación o afirmación del derecho internacional: ¿qué se niega o qué se afirma en torno al derecho de gentes? Walz advierte sobre la necesidad de la precisión en este punto para saber qué autores se encuentran dentro de una y otra categoría. Siguiendo su criterio, se puede entender que un negador del derecho internacional es aquel que no admite su juridicidad, comprendiéndose así a una serie de pensadores que tienen como punto en común "el excluir al llamado derecho internacional del reino de los valores jurídicos” (Walz 1943: p. 34).

Esta precisión inicial resulta ser de suma importancia porque el criterio walziano no asume que un negador no reconozca "la existencia del complejo normativo constituido por el derecho internacional", sino que su negación reside en descartar "simplemente el carácter jurídico de este complejo de normas" (Walz 1943: p. 35). Este matiz es fundamental porque como veremos luego, Bello no niega la presencia de normas en el derecho de gentes, sino que no les reconoce, realmente, fuerza jurídica. Este elemento se agrava aún más si se tiene en mente que el libro Principios de Derecho Internacional fue considerada "la primera obra científica de una utilidad general é [sic] incuestionable, que se ha dado á [sic] luz en la América Española"2 (Bello 1873: p. 5).

En tanto que la finalidad del presente trabajo es comparar las posturas de Silva Santisteban y Bello como ejemplos en nuestra región, respectivamente, de un defensor del

\footnotetext{
${ }^{1}$ Los tratados de la Paz de Westfalia fueron celebrados en 1648, en las ciudades de Osnabrück y Münster, para darle fin a la Guerra de los Treinta Años. El primer tratado fue celebrado entre Suecia, por un lado, y, por otro lado, el Sacro Imperio Romano Germánico. El segundo tratado tuvo como partes a Francia y al referido imperio. Es preciso entender, en este punto, que sostener que tales tratados dan origen al derecho internacional es una consideración historiográfica de los iusinternacionalistas, por lo que no significa que el Estado moderno haya surgido a partir de tales tratados. (Neff 2014: pp. 139 - 141).

${ }^{2}$ Esta afirmación aparece en la nota del libro de Bello y que fuera escrito por Antonio José de Irisarri, bajo el título de "Advertencia".
} 
derecho de gentes y un negador del mismo, será preciso presentar quiénes los precedieron en este debate. En ese sentido, aunque se parte de la identificación de Francisco de Vitoria ${ }^{3}$ y de Hobbes como ejemplos respectivamente de la opción ibérica y opción inglesa en el sentido morsiano, también pueden ser considerados representantes de la dicotomía generada entre la defensa y negación del derecho internacional. En este punto, se analizarán a estos autores en la lógica de la defensa y cuestionamiento del derecho internacional y, en la siguiente sección, la configuración de la opción ibérica y la opción inglesa, desde la visión de Morse y los aportes de Becker Lorca.

Francisco de Vitoria es, innegablemente, el padre del derecho internacional ${ }^{4}$, conjuntamente con Grocio. La paternidad del primero reside, esencialmente, en dos grandes obras que son la Relectio des indis y la Relectio de potestate civili ${ }^{5}$, aunque ninguno de estos escritos constituyan propiamente una sistematización del derecho internacional (Gómez Robledo 1989: p. 114; Anghie 1996: p. 321).

La primera es una obra en la que Vitoria busca justificar la empresa de colonización de la Corona Española en las nuevas Indias, enfrentándose, fundamentalmente, a dos tesis sostenidas en su época, a las cuales se recurría con el mismo propósito. Por un lado, a la autoridad del Papa y, por otro lado, a la idea del ius inventionis. La primera la rechazó con base en el argumento que los indios, lejanos geográficamente de Europa, no conocían la fe cristiana y, por ello, no era posible utilizar esta autoridad como posible elemento de justificación ${ }^{6}$. La segunda tampoco la admitió, debido a que no consideraba que los nuevos parajes fueran tierras sin dueño ${ }^{7}$, como se sostenía con el ius inventionis, por el cual se postulaba que las tierras descubiertas eran res nullius.

Esta labor de encontrar la causa justa de la conquista española es una de las razones por las cuales a Vitoria se le confiere el título de padre de derecho internacional, puesto que admite la posibilidad de un relacionamiento político y comercial más allá de Europa con otras entidades políticas que, más adelante, conformarían también la comunidad del totius orbis, idea desarrollada con maestría en la Relectio de potestate civili, bajo los siguientes términos:

"Que el derecho de gentes no solo tiene fuerza por el pacto o acuerdo entre los hombres, sino que tiene fuerza de ley. En efecto, el orbe todo, que en cierto modo constituye una única república, tiene el poder de promulgar leyes justas y convenientes para todos, cuales son las del derecho de gentes. De donde se sigue que pecan mortalmente quienes violan el derecho de gentes, ya sea en la paz o en la guerra, o en los asuntos más graves, como es el no respetar a los legados. No le es lícito a un reino particular no querer atenerse al

\footnotetext{
${ }^{3}$ Francisco de Vitoria (1483 - 1546) fue un fraile dominico, de origen español, considerado el mayor representante de la Escuela de Salamanca (Brett 2012).

${ }^{4}$ También ha recibido el apelativo de "padre del derecho colonial moderno" (Iannarone 1970: p. 5).

${ }^{5}$ Gómez Robledo agrega la Relectio de temperantia como uno de esos textos fundantes; sin embargo, el análisis que realiza el profesor mexicano se centra en un fragmento de esta relección vitoriana que se critica en su autenticidad (1989: p. 22). Iannarone ha considerado esta Relectio como un texto antecesor al de Relectio des indis (1970: p. $18)$.

${ }^{6}$ Como señala Vitoria, “[...] no tienen por qué acatar la sentencia del Papa quienes no sean cristianos, pues que por ningún otro título puede el Papa condenar o castigar, si no es por el de ser Vicario de Cristo" (1975: p. 82).

${ }^{7}$ En tal sentido, Vitoria sostiene que "[e]s de derecho de gentes que se concedan al ocupante las cosas que no son de nadie (...); pero como aquellos bienes no carecían de dueño, no pueden ser comprendidos por este título" (1975: p. $69)$.
} 
derecho de gentes, ya que ha sido promulgado por la autoridad del orbe entero" (Vitoria 2008: p. 63)

Este párrafo inscrito en el punto 21 de la citada Relectio, con el título "Si las leyes civiles obligan a los legisladores y, en máximo grado, a los reyes. Vigencia del Derecho de gentes", ha sido considerado por autores de la talla de Gómez Robledo (1989: pp. 32-33) y Miaja de la Muela (1965: p. 355) como un pasaje clave para la comprensión del pensamiento vitoriano. Es a partir de este texto que se puede sostener que Vitoria reconoce que el derecho de gentes es uno que tiene carácter jurídico ("fuerza de ley") y que, además, debe ser respetado en todo el orbe. Esta inspiración, si bien inscrita en el marco de la lógica del derecho natural, resulta ser la pieza clave bajo la cual se construirá, posteriormente, el pensamiento de Grocio quien también defenderá el carácter jurídico y aplicable del derecho internacional.

En contra de esta posición que defiende el ethos jurídico del derecho internacional, se encuentra una serie de autores, de los cuales hemos escogido a Hobbes ${ }^{8}$, que puede ser apreciado como "el primero que en la doctrina haya negado seriamente el carácter jurídico del derecho internacional" (Walz 1943: p. 39; en la misma línea, Truyol y Serra, 1998: pp. 86-87), cuyo pensamiento se resume en "El Leviatán" $(1651)^{9}$ y cuyas premisas son compatibles con las de otros negadores importantes del derecho internacional, como Austin ${ }^{10}$, en el sentido que el primero sostendrá que, dada la ausencia de un gobierno internacional, no sería posible afirmar que el derecho internacional sea, realmente, Derecho (Orakhelashvili 2011: p. 8).

Hobbes distingue entre un "estado de naturaleza", caracterizado por un status quo de enfrentamiento total ("bellum omnium contra omnes") 11 , y "un estado de sociedad". Para superar el primer estado de cosas, que es una situación completa de anomia de carácter hipotético (no necesariamente histórico), y llegar al segundo estado, es fundamental un contrato social. En ese sentido, Hobbes es, al igual que Locke y Rousseau, un teórico del contrato social (José Contreras 2016: p. 170), solo que sostendrá, a diferencia de estos filósofos, la preexistencia de un "estado de naturaleza" caótico, al cual lo denominará "guerra" (Hobbes 1998: p. 84), no en el entendido de un enfrentamiento bélico, sino en la "disposición manifiesta a ella durante todo el tiempo en que no existe seguridad de lo contrario"12. En ese sentido, Hobbes no comparte la idea de sociabilidad $^{13}$ que sostiene Aristóteles, concepto clave en el pensamiento vitoriano.

${ }^{8}$ A Hobbes (1588 - 1679) se le considera, junto con John Locke, como uno de los pensadores ingleses más importantes del siglo XVII (José Contreras 2016: p. 169).

${ }^{9}$ Para efectos del presente trabajo se ha revisado la edición en inglés de Oxford, a cargo de Gaskin (1998).

${ }^{10}$ Según Austin, el derecho internacional carece de las características del derecho positivo, porque sus normas no son establecidas por un poder supremo y porque estas no tienen el carácter de mandato (Murphy 2012, p. 6; Walz 1943: p. 119).

${ }^{11}$ Hobbes utiliza la expresión "as is of every man, against every man" (1998: p. 84).

${ }^{12}$ En este punto, Hobbes señala "known disposition thereto, during all the time there is no assurance to the contrary" (1998: 84).

${ }^{13}$ La sociabilidad aristotélica se sustenta en el libro "Politeia" (Política), en el cual el Estagirita sostiene que "[...] la polis existe en la naturaleza, y que el hombre es por naturaleza un animal político. Quien, pues, por su naturaleza - y no por accidente - [carece] de polis es o un loco, o un [ser] superior, o un individuo como [aquel] a quien condena Homero, [como alguien] sin familia, sin ley y sin hogar" (Aristóteles 1989: pp. 135 - 136). A esta apreciación de la naturaleza humana, Hobbes se opondrá a partir de la tesis Homō hominì lupus est, que no comienza con "El Leviatán", sino con una obra anterior del mismo autor inglés, "De Cive". En efecto en este 
De un escenario como el del "estado de naturaleza", se denegará, no solo la premisa aristotélica de la sociabilidad, sino sobre todo la posibilidad de la justicia. Por ello, sostendrá que "[d] e esta guerra de todos contra todos se sigue [...] que nada puede ser injusto. [...] Donde no hay poder común, no hay ley; donde no hay ley, no hay injusticia"14 (Hobbes 1998: p. 85).

Si bien Hobbes no aporta ninguna teoría profunda sobre el derecho internacional, en la línea de esta premisa de negación del derecho y de la justicia, llegará a sostener lo siguiente:

"Con respecto a los cargos de un soberano a otro, que están comprendidos en esa ley, que comúnmente se llama la ley de las naciones, no necesito decir nada en este lugar; porque la ley de las naciones y la ley de la naturaleza es lo mismo. Y cada soberano tiene el mismo derecho, de procurar la seguridad de su pueblo, que cualquier hombre en particular puede tener, de procurar su propia seguridad. Y la misma ley, que dicta a los hombres que no tienen un gobierno civil, lo que deben hacer y lo que deben evitarse unos a otros, dicta lo mismo a las comunidades, es decir, a las conciencias de los príncipes soberanos y las asambleas soberanas." 15 (Hobbes 1998: p. 235)

Asumiendo la identificación del derecho de gentes con el derecho natural, Hobbes tiene como postura que cada Estado debe buscar protegerse a sí mismo, puesto que no existe para él una autoridad por encima de los Estados que brinde esa seguridad y que, por lo tanto, pueda fundamentar el derecho internacional positivo.

En este último detalle veremos, posteriormente, una conexión entre la postura de Bello con la de Hobbes, porque este autor es el primero en sostener que la preocupación de cada ser humano y, del mismo modo, de cada sociedad, es la de su propio bienestar. En ese sentido, no solo ofrece una negación del derecho internacional, sino que reconoce que la manera cómo se desenvuelven los Estados en el escenario internacional es buscando un aumento de su poder (Walz 1943: p. 47).

Otro autor importante a tomar en cuenta, para comprender a Bello, es Jeremy Bentham, famoso pensador considerado padre del utilitarismo inglés. Este filósofo sostendría que la ley es susceptible de ser modificada y que, en ese camino, debe generar el máximo nivel de felicidad para la cantidad más amplia de personas. Si bien se inclinaría por aceptar la prevalencia del interés público por sobre el interés privado, no reconocería la existencia de los derechos naturales y se opondría, por ello, a la Déclaration des droits de l'homme (1789) pronunciada en la

último libro, Hobbes señala "To speak impartially, both sayings are very true; That Man to Man is a kind of God; and that Man to Man is an arrant Wolfe" (1987: p. 24).

${ }^{14}$ En su version en inglés, se afirma lo siguiente: "To this war of every man against every man, this also is that nothing can be unjust. The notions of right and nothing is wrong, justice and injustice have there no place. Where there is no common power, there is no law: where no law, no injustice."

${ }^{15} \mathrm{La}$ version original de la cita dice lo siguiente: "Concerning the offices of one sovereign to another, which are comprehended in that law, which is commonly called the law of nations, I need not say anything in this place; because the law of nations, and the law of nature, is the same thing. And every sovereign hath the same right, in procuring the safety of his people, that any particular man can have, in procuring his own safety. And the same law, that dictateth to men that have no civil government, what they ought to do, and what to avoid in regard of one another, dictateth the same to commonwealths, that is, to the consciences of sovereign princes and sovereign assemblies." (Hobbes 1998: p. 235) 
Revolución Francesa, al considerarla como un terrible invento metafísico (Reale, G.; Antiseri, D.; 1988: pp. 286-288). Respecto al derecho internacional, Bentham argüía que la fuerza de un tratado entre dos naciones es menor al vigor de un contrato entre dos individuos, lo cual demuestra su inclinación por minusvalorar el valor jurídico del derecho de gentes (Orakhelashvili 2011: p. 10).

Antes de ingresar a examinar las posturas de Santisteban y de Bello, entraremos a ofrecer un marco teórico que permita discurrir mejor en la discusión que se ha pretendido plantear aquí.

\section{La era decimonónica y el derecho de gentes a la luz de la postura de Morse}

El siglo XIX es un periodo de la historia del derecho de gentes que se caracteriza, a diferencia de los siglos anteriores, por experimentar la universalización del derecho internacional, discutiéndose básicamente dos tesis de cómo se produjo tal universalización (Becker Lorca 2010: pp. 475-477) ${ }^{16}$ : La primera tesis, que podemos llamar tradicionalista, sostiene que tal universalización es producto de la expansión del ius publicum europeum, que se desarrolló desde el siglo XVII con los tratados de la Paz de Westfalia, así como de la superposición de esta versión del derecho internacional sobre las otras versiones posibles de este en otros lugares del orbe. La segunda tesis se distancia de la anterior, planteando que tal universalización no supuso un proceso de simple recepción del derecho internacional desarrollado por Europa, sino una asimilación estratégica de sus instituciones y su replanteamiento por las potencias extraeuropeas en pro de sus intereses nacionales, entre las cuales destacan las de Hispanoamérica, recién independizadas.

En el primer caso, la tesis tradicionalista es una que parte de una construcción desigual de las relaciones internacionales, en la que las potencias europeas se presentan como aquellas que ostentan un mayor nivel de civilización, frente a las realidades extraeuropeas, a las que se les concibe como primitivas, salvo ciertas excepciones ${ }^{17}$ (Cassese 2013: pp. 34-37). En cambio, la tesis cuestionadora se inclina por concebir a tales potencias fuera de Europa como capaces de asimilar, deconstruir y replantear, hasta cierto punto, el derecho internacional recibido.

Ante ambas posiciones, existen razones para inclinarse por la tesis cuestionadora. En primer lugar, la tesis tradicionalista estudia el proceso desde Europa y, por ello, bajo los intereses historiográficos europeos. Esto puede implicar una distorsión en la comprensión de las realidades de las Américas porque no se percibe a las potencias hispanoamericanas bajo un rol activo o simplemente se les ignora. La tesis tradicional, en segundo lugar, omite por desinformación lo

\footnotetext{
16 Esta universalización tiene su explicación en las características del siglo XIX, donde la movilidad humana y del capital se dio como nunca antes en la historia. Como destaca Osterhammel, "[t]oda la historia [del siglo XIX] desborda de movimiento: viajes, pueblos que migran, campañas militares, comercio con lugares remotos, difusión de religiones, lenguajes y estilos artísticos". Asimismo, señala que el "capital fluyó por todo el globo impulsado en particular por la instalación de múltiples redes ferroviarias, más que por la economía fabril” (2015: pp. 1274 1275).

${ }^{17}$ En el marco del derecho internacional clásico, las relaciones con las potencias extraeuropeas se construían de dos maneras. Por un lado, se celebra capitulaciones con potencias orientales y asiáticas. Por otro lado, a aquellas estructuras que no se les consideraba, desde la óptica europea, suficientemente evolucionadas, se les colonizaba (Cassese 2013: pp. 34-37).
} 
que ocurre en las latitudes extraeuropeas y también desconoce que, fuera de Europa, hubo también producción literaria en derecho internacional.

El presente estudio parte de aceptar la tesis cuestionadora porque no se puede apreciar, en su verdadera dimensión, las contribuciones de dos autores como Silva Santisteban y Bello, si se sigue asumiendo la tesis tradicional que se limita a los aportes de pensadores que hayan nacido en Europa o que hayan producido su bibliografía en esas latitudes. Aquí, igualmente, cabe precisar un matiz. No se trata de rechazar el hecho que, durante el siglo XIX, Europa haya influido en las concepciones de los ius internacionalistas locales; por el contrario, al igual que con Morse, se coincide en que la tradición del pensamiento europeo se transmite o se traslada, pero este es el telón de fondo del cual se parte, sin que ello agote la riqueza inventiva de los pensadores del derecho de gentes en nuestro continente. Concebir que se produjo solo la recepción de las teorías europeas del derecho internacional es, básicamente, caer en el error de sobrevalorar lo europeo e infravalorar los aportes extraeuropeos y, en este caso, de la América Hispánica.

Este proceso de replanteamiento o reacomodo de la herencia cultural recibida, sin que ello implique propiamente una innovación completa o un olvido de todo el tesoro cultural prefabricado, es uno que se puede percibir en varias de las contribuciones de los autores del derecho de gentes de la época. Son ellos los principales artífices de esta labor de asunción del ius publicum europeum y también los que empiezan a remodelar esta herencia desde su visión periférica.

Es bajo este esquema que, cada vez más, se vuelve necesaria la búsqueda de ius internacionalistas decimonónicos en nuestra región, por razones de índole científica e histórica, porque la falta de estudios sobre esta materia es un perjuicio a los orígenes regionales del derecho de gentes. Por una parte, porque es una etapa muy poco estudiada en general y de la que se llega a asumir automáticamente la posición eurocéntrica, cuando es posible también percibir que los actores periféricos y semiperiféricos no son para nada pasivos ante el tesoro cultural heredado. Por ello, resulta de sumo interesante saber cómo es que, en la realidad hispanoamericana, es posible sostener tal papel activo de los autores locales de derecho de gentes (Becker Lorca 2010: pp. 477-479).

En la construcción de ese papel activo, es preciso indicar que la reacción de los autores que estudiaron el derecho de gentes no tuvieron necesariamente las mismas visiones, sino que intentaron construir sus propias posturas. Esto se debe a que, frente a un mismo fenómeno, nunca existe una sola aproximación: es más que probable que cada autor pudiera tener una visión que contara, más allá de puntos en común, con particularidades que los caracterizaron y que merecen ser estudiadas.

Justamente, para resolver el cuestionamiento trazado en esta investigación es necesario acudir al planteamiento de Morse en su obra magna $O$ espelho de Próspero: cultura e idéias nas Américas (1988). Este libro llama la atención de que el siglo XIX no comenzó sin una etapa de formación previa, por lo que para la explicación de cómo es que se ha llegado a la exportación del derecho de gentes y su asimilación/cuestionamiento en nuestra región, es necesario verificar, a grandes rasgos, los pasos que preceden a este siglo; más aún si se busca entender cómo es que los autores del derecho de gentes no reaccionaban necesariamente de la misma manera ante un universo jurídico todavía novísimo para su propia realidad. 
Morse sostiene que existió, antes de su separación, una matriz cultural e intelectual común de origen europeo entre Hispanoamérica y Angloamérica (1988: p. 22). Esta etapa se inició para este pensador en el siglo XII y evolucionó hasta el siglo XVII, produciéndose progresivamente una bifurcación de tradiciones que darán la impronta de los pensamientos filosóficos, jurídicos y políticos de estas dos realidades. Pero como advierte este autor, no fueron modelos herméticos, ni realidades que carecieran de contradicciones internas (Morse 1988: p. 22).

La diferencia entre la opción hispánica del siglo XVI, que influirá con mayor fuerza en Hispanoamérica, y la opción inglesa, cuyo acervo se transmitirá en el siglo XVII a Angloamérica, puede graficarse en las diferencias respectivas de dos autores clásicos como Francisco de Vitoria y Thomas Hobbes (Morse 1988: pp. 60-63).

Morse anota algunas diferencias notorias en las posiciones de ambos pensadores, de las cuales cabe resaltar aquí algunas. Para el caso de Vitoria, este se dedicó a analizar las justificaciones de la expansión del imperio español y la colonización de las Indias en el marco, principalmente, del derecho natural (Anghie 1996: pp-323-327); en cambio, Hobbes buscó, sobre todo, la reconstrucción de su propia sociedad para contrarrestar su caos originario. Mientras que Vitoria procuró un esquema pensado en abstracto, en una sociedad común o general, Hobbes se inclinó por una sociedad en la que prima lo particular o individual. Siguiendo esta inclinación por la colectividad en el pensamiento vitoriano, el gran representante de la Escuela de Salamanca prefirió construir una teoría política del bien común; en cambio, Hobbes se manifestó a favor de un Estado que pudiera garantizar los derechos y libertades de cada ciudadano.

Ya para el siglo en que se celebró la Paz de Westfalia, se habrían configurado las dos tradiciones diferenciadas y que podemos ver como tipos ideales, en la terminología weberiana ${ }^{18}$. Pero aquí debemos precisar que, una vez diferenciadas las tradiciones hispánica e inglesa, en el siglo XIX, estas van a desembocar en una mixtura que se alejará de la diferenciación notoria que podemos hallar en Vitoria y Hobbes. De la tradición común en el siglo XII se pasó, entonces, al distanciamiento en el siglo XVII y luego, una vez separadas, a un acercamiento que terminará en una mixtura de enfoques en pleno siglo XIX. De este modo, en la propia Hispanoamérica va a ser posible encontrar un panorama que ya no se cierra a una visión únicamente vitoriana o iusnaturalista, sino también va a observarse la presencia de la otra tradición empirista que se va introduciendo y que va siendo parte del repertorio ideológico de ciertos pensadores.

Y es aquí donde se debe resaltar la presencia de los dos grandes autores que se pretenden estudiar. Silva Santisteban y Bello son hispanoamericanos, uno de nacionalidad peruana y el otro venezolano, respectivamente, pero entre ellos no es posible encontrar una suerte de "vitorianismo puro". Por el contrario, se perciben ciertos distanciamientos o reparos con el propio iusnaturalismo, de manera mucho más notoria en la obra de Bello.

\footnotetext{
18 Weber en La ética protestante y el espíritu del capitalismo ha utilizado la noción del tipo ideal cuando trata de explicar los rasgos del calvinismo, el pietismo, el metodismo y el movimiento baptista. En ese contexto, señala que, en su obra, se exponen "las ideas religiosas en una estructura de «tipo ideal» que rara vez se da en la realidad. Pues, precisamente porque es imposible trazar fronteras claras en la realidad histórica, sólo podemos esperar dar con sus efectos específicos investigando sus formas más estructuradas.” (2016: p. 143). El tipo ideal sería, entonces, una unidad modélica que se contrasta con la realidad, destinada a explicar un determinado fenómeno histórico (Sánchez de Puerta Trujillo 2006: pp. 15 - 19).
} 
Este artículo pretende encontrar, en los aportes de las principales obras de estos grandes autores de nuestra región, qué tanto es posible partir de la tesis de Morse para explicar la diferenciación de sus obras en la lógica de las tradiciones neotomista, para efectos de entender a Silva Santisteban, y empírica, en el caso de Bello. Comenzaremos, a continuación, explicando el planteamiento del primero.

\subsection{La concepción de Silva Santisteban sobre derecho de gentes}

José Silva Santisteban ha sido considerado como uno de los primeros principales internacionalistas peruanos en el siglo XIX (Basadre Ayulo 2001: p. 474), a quien luego le seguiría Pablo Pradier-Fodéré y Carlos Wiesse en importancia. La obra del primero en materia de derecho internacional público, y que ha quedado para la posteridad, es la del Curso de derecho internacional o de gentes ${ }^{19}$, a la cual el propio autor califica de un "curso sencillo y metódico" 20 (1858: p. 43), cuyo propósito se destina a una comprensión del derecho de gentes, desde la filosofía y la historia de esta rama (Silva Santisteban 1858: pp. 19 -20), debiéndose esta doble percepción a que "(...) al lado de la historia de los hechos, deberá ir la de la ciencia, para conocer su marcha progresiva, en armonía con el desarrollo de las instituciones" (1858: p. 20). En el fondo, Silva Santisteban quiere lograr la presentación del derecho de gentes desde una mirada científica de la misma y esta es la clave que explica todo el libro. Por eso, llega a afirmar que la “(..) ciencia de Grocio (...) no se halla elevada aún a la altura de verdadera ciencia” (1858: p. $42)$.

Con el propósito de entender la concepción de Silva Santisteban, y bajo la lógica del debate planteado entre afirmadores y negadores del derecho internacional, en este acápite se estudiará su noción de derecho de gentes y los fundamentos filosóficos de los cuales parte. Este análisis es uno que no pretende agotar toda la sabiduría expuesta por el profesor peruano, sino que busca presentar elementos que permitan visualizar la tesis de Morse para efectos de la evolución del derecho de gentes en el siglo XIX.

Silva Santisteban (1858: p. 57) define al derecho internacional, del modo siguiente:

"la ciencia que trata de arreglar las relaciones internacionales según los principios eternos de justicia, o en otros términos, establecer los principios a que deben sujetarse las naciones en la prestación de medios externos necesarios para alcanzar sus fines racionales" ${ }^{21}$.

\footnotetext{
${ }^{19}$ Basadre Ayulo destaca también de este autor el libro Derecho peruano. Parte Civil de 1853 (2001: p. 475).

${ }^{20}$ El libro de Silva Santisteban (1858) se compone de una introducción (pp. 19 - 20); un primer período (21-28) y un segundo período (pp. 29 - 46) en la evolución del Derecho de gentes; una parte general, titulada “Ciencia” (pp. 47 - 72); y dos partes especiales referidas al derecho positivo; la primera titulada "De las naciones consideradas en su estado normal o de paz" (pp. 73 - 164), y la segunda "De las naciones en un estado anormal o de guerra (pp. 165 221).

${ }^{21}$ Actualmente, la cuestión relativa a la definición del derecho internacional no es que se encuentre resuelta (Rosales Zamora 2018: pp. 16-21), pero es importante aquí hacer mención a que autores del prestigio de Daillier, Forteau y Pellet han preferido definir al derecho internacional como derecho aplicable a la sociedad internacional (2009: p. 43). Esta definición busca no solo responder al principio básico ubi societas, ibi ius, sino sostener que el derecho internacional es, como tal, un sistema jurídico, evitando así una construcción conceptual que limite materialmente tanto los elementos de ese derecho -evitando dar una respuesta definitiva a si este derecho comprende solo a las
} 
Resulta bastante interesante que este autor califique al derecho internacional como ciencia, destacando de manera muy evidente el atributo racional que esta rama del derecho tendría. Como desmiente Morse (1988: p. 34 - 36), la postura neotomista nunca cuestionó la cientificidad del conocimiento, por el contrario, resistió a aquellos autores que introducían los planteamientos conjeturales o probabilísticos. Esta tesis responde, en el fondo, al intento de aquel profesor peruano de sostener el carácter científico del derecho internacional, haciendo frente a la postura empírica, que negaba tal estatus. En efecto, critica a "los que se limitan a exponer los usos actuales y juzgan que no existe un derecho de gentes, porque no hay leyes impuestas a las naciones ni autoridad superior de donde emanen, [confundiendo] el fondo con la forma, [pretendiendo] resolver cuestiones de alta filosofía con las ideas vulgares y empíricas de derecho" (Silva Santisteban 1858: 57).

En ese sentido, el profesor peruano abraza el racionalismo y concibe como el peor enemigo del cuestionamiento de la cientificidad del derecho internacional al empirismo. Pero aquí, al inclinarse por el racionalismo es que Silva Santisteban está llevando el debate al punto explorado por Morse, puesto que este uso de la razón que pregona en el carácter científico del derecho de gentes es uno que se orienta por los "principios eternos de justicia", lo cual revela una influencia neotomista que se trasluce en la idea de la "existencia del principio que debe presidir en las relaciones internacionales, o de una ley de las naciones, [que] se deduce pues lógicamente de la naturaleza misma de la nación"22 (Silva Santisteban 1858: p. 55).

Uniendo ambos elementos, es posible entender que Silva Santisteban sea un iusnaturalista racionalista - superando a Vitoria, pero no alejándose de este -, que pretende sostener que, más allá del caos de las relaciones internacionales, existen principios que trascienden la voluntad de las naciones. Y que es intrínseco a esta unidad política que no se aísle, sino que se relacione, en virtud del principio de sociabilidad, el cual supone el estrechamiento de las relaciones exteriores con las otras naciones, para configurar "en una sola familia la humanidad entera" (Silva Santisteban 1858: p. 54).

Este iusnaturalismo racionalista es uno que descansa en la admiración del autor por Grocio, al cual lo concibe como verdadero científico del derecho internacional. Y este punto es trascendental porque Grocio es continuador de Vitoria, como lo sostiene el profesor mexicano Gómez Robledo (1989: 101 y ss.), al tratar de resolver el dilema relativo de quién de los dos es padre del derecho internacional. Y la respuesta del tratadista mexicano es de una sabiduría admirable porque encuentra que ambos lo son, sin desmerecer sus aportes, solo que el descubridor es Vitoria con sus ideas del totius orbis y del ius communicationis, correspondiéndole a Grocio el papel de sistematizador de este último principio en el famoso Mare liberum. Así lo admite Van der Vlugt, como cita Gómez Robledo, al sostener el primero

\footnotetext{
normas y no a los principios, si este derecho puede tener manifestaciones no mandatorias como el soft law, entre otras cuestiones-, así como la posibilidad de la aparición de nuevos sujetos de derecho internacional.

22 Aquí debe precisarse que este autor utiliza el término "nación" para referirse a lo que actualmente el derecho internacional concibe como Estado, cuando sostiene que el término designa a una "comunidad de origen o nacimiento, de donde resulta que a los individuos de una raza, a los descendientes de un padre común, se les califica de esta manera" y cuyo "principio dominante es la unidad política, y no solo intrínseca sino representativa, es decir, que la nación sea representada como una unidad en sus relaciones con las demás" (Silva Santisteban 1858: p. 53).
} 
que "[e]ste opúsculo no tiene otro valor que el de un estudio bien hecho de segunda mano, importado de la sabiduría española" (1989: p. 113).

Sintiéndose heredero de Grocio, Silva Santisteban es sino un continuador de Vitoria, y con ello hace suyo el planteamiento iusnaturalista del neotomismo en la explicación del enfoque morsiano, rechazando la visión empirista que desciende en un utilitarismo, como veremos con el desarrollo de las ideas de Andrés Bello.

\subsection{Andrés Bello y Principios del Derecho Internacional}

Este personaje no excepto de controversia ha sido considerado, no obstante, como uno de los mayores intelectuales del siglo XIX en las Américas ${ }^{23}$. De origen caraqueño, estudió Derecho en Londres y se encargó de ordenar los manuscritos del mayor representante del utilitarismo, Jeremy Bentham, a quien conoció personalmente (Otero Parga 2017: p. 86). Ocupó luego diversos cargos públicos, entre los cuales destacó el puesto de oficial mayor del Ministerio de Hacienda de Chile (1834) y el cargo de senador de la República (1832 a 1864). De una actividad intelectual muy prolífica, Bello regentó el Colegio de Santiago hasta 1832; cuando esta institución se clausuró, Bello se dedicó a brindar, en su casa, lecciones de diversas materias, entre las cuales precísase mencionar la de derecho de gentes. De 1843, en adelante, dirigiría, en calidad de rector, la Universidad de Chile hasta su muerte (Olano García 2007: pp. 70 - 72).

La obra cumbre de Bello en el terreno del derecho internacional fue el famoso libro Principios de derecho internacional ${ }^{24}$, que, originalmente, se denominó Principios del derecho de gentes, en su primera publicación de 1832. Este texto alcanzará un gran éxito a nivel de la región en el siglo XIX por el esfuerzo desplegado por Bello de leer, en palabras de un exaltado Antonio José de Irisarri, “[...] todos los libros que deben componer la biblioteca del hombre que quiere conocer á [sic] fondo el derecho internacional" 25 .

En las "Nociones preliminares" de su obra, es que Bello desarrollará la noción de derecho de gentes, bajo la definición de "colección de las leyes ó [sic] reglas generales de conducta que las naciones ó [sic] Estados deben observar entre sí para su seguridad y bienestar común" (1873: p.11).

Debe llamarse, especialmente, la atención sobre esta definición propuesta y que se mantiene a lo largo de las reediciones del libro citado, porque esta es una de las principales razones para considerar a Bello como un negador del derecho internacional. Define al derecho de gentes como una "colección", que podría entenderse en el sentido de "un conjunto ordenado de cosas, por lo común de una misma clase y reunidas por su especial interés y valor" ${ }^{26}$. Este carácter de conjunto que propone Bello supone una mirada específica del derecho de gentes, no

${ }^{23}$ Como opina Otero Parga, "sabemos también que Bello estudió en profundidad latín y que tuvo una cultura muy [v]asta para su tiempo." (2017: p. 94).

${ }^{24}$ La obra Principios de Derecho Internacional se compone de unas "Nociones preliminares" (pp. 19 - 22) y de tres partes, referidas al estado de paz (pp. 23-146), estado de guerra (pp. 147- 304) y los derechos y funciones de los agentes diplomáticos (pp. 305 al 323).

${ }^{25}$ Como se ha hecho notar, anteriormente, esta afirmación pertenece a Antonio José de Irisarri, y se encuentra en el libro de Bello que trabajamos aquí, bajo el título de "Advertencia".

${ }^{26}$ Esta es la definición de colección, en su primera acepción, que da el Diccionario de la lengua española. 
en el sentido de que conforme un sistema jurídico cohesionado, sino que configuraría un simple listado de reglas. Ante ello, debe recordarse que se erige una evidente distancia entre señalar que es una rama del Derecho o un sistema jurídico, a afirmar, simplemente, que es una colección de leyes o reglas generales de conducta.

Este elemento de apreciar al derecho internacional como una colección supone que Bello concebía al derecho de gentes, no tanto desde una preocupación científica, sino más bien como una arista más del saber. Recuérdese que el intelectual caraqueño fue un humanista que se inclinó por más de una rama del conocimiento y creo que, por muy sabia y cara que haya sido la construcción de su libro, esta también recibe el influjo de su tiempo y, más aún, el proceso de formación de su autor.

Un segundo elemento a tomar en cuenta es que Bello no solo opta por inclinarse a definir el derecho de gentes como una colección, sino que es una colección de leyes o reglas generales, presentando con ello una inclinación o preferencia por una lectura positivista del Derecho. En ese sentido, también indica, en este capítulo que "[...] las reglas establecidas por la razón ó [sic] por el consentimiento mutuo, son las únicas que sirven, [...] para el ajuste de las diferencias entre soberanos" (Bello 1873: p. 19).

En ese sentido, reconoce un derecho de gentes natural, que vendría a ser el que surge de la razón; y un derecho de gentes positivo, que es el convencional, sea expreso o tácito, y "cuya fuerza solo se deriva mediatamente de la razón, que prescribe á [sic] las naciones, como regla de importancia suprema, la inviolabilidad de los pactos" (Bello 1873: p. 16). Sea uno u otro tipo de Derecho lo entiende ligado, siempre, a la razón.

Pero este racionalismo lo lleva a un sendero distinto que al de Silva Santisteban, dado que Bello afirmará que "[n]o hay un código en que estén recopilados los preceptos o prohibiciones del Derecho internacional, sea natural, sea instituido, lo que produce incertidumbres y dudas, que los Estados poderosos no dejan nunca de interpretar a su favor" (1873: p. 19). Esta es una lectura que demuestra la clara noción del autor por un reconocimiento del estado de guerra hobbesiano al emplear una lectura de las relaciones internacionales desde el poder.

En ese sentido, es importante comprender que hay un nexo fundamental entre la concepción del derecho de gentes como colección con la formación londinense de Bello, instrucción que, como se mencionó hace unas líneas atrás, fue en Derecho. En ese sentido, Bello había bebido directamente del espíritu empirista inglés y más aún debe haber sentido una especial cercanía a la obra de Bentham, porque de otra forma, no se hubiera dedicado a ordenar su obra. En ese sentido, Bello ha debido conocer el derecho internacional bajo el influjo del espíritu empirista y utilitarista, lo cual refuerza la propuesta de abordaje que hemos presentado ${ }^{27}$.

\footnotetext{
${ }^{27}$ Algunos autores se han inclinado en considerar a Bello como un pensador que recibió una fuerte influencia de Suárez, un importante pensador del derecho internacional y representante, al igual que Vitoria, de la Escuela de Salamanca (Otero Parga 2017: pp. 93-98). Se debe, gentilmente, discrepar de esta estimación. Si bien es más que probable que Bello haya conocido de la obra de pensadores de la talla de Grocio o de Suárez, esto no implica un real cambio en su concepción relativa a la definición de derecho internacional. Esta tesis se confirma con el hecho que a lo largo de las tres ediciones de su texto Principios de derecho internacional, el intelectual caraqueño no haya alterado el concepto de derecho internacional como listado de leyes, acercándose a la aproximación empirista y utilitarista que se ha identificado.
} 
En suma, la definición de derecho de gentes por la que opta Bello demuestra encajar, en este terreno, con la presentación de la opción inglesa que propone el texto de Morse.

\section{Contraste de las posturas de Silva Santisteban y Bello bajo el esquema de Morse}

Como se ha visto, el debate de los afirmadores y negadores del derecho internacional tiene también sus representantes a nivel de la literatura hispanoparlante, producida en nuestra región durante el siglo XIX. Si bien este debate in concreto no es planteado por Morse, este autor cuenta con una lucidez extraordinaria que permite explorar el encuentro de dos gigantes del derecho de gentes en nuestro continente, que son Silva Santisteban y Bello, los cuales además tienen el mérito de ser los primeros autores que tratan sobre la materia de un Derecho que va más allá de las fronteras, mostrándose, a su vez, como herederos de tradiciones ideológicas diferentes entre sí.

En efecto, lo que se sostiene es que tales herencias intelectuales son las que definen que uno y otro autor acepten o no la juridicidad del derecho internacional.

Morse ilustró los contrastes entre la opción hispánica y la opción inglesa en un sentido político e intelectual, como las grandes matrices en Europa que explicarán, posteriormente, las dinámicas intelectuales, políticas y económicas de las colonias hispánicas en nuestro continente, en el primer caso, y las colonias británicas, en el caso de Estados Unidos, así como de su gesta independiente y su posterior destino. Tales modelos no se encierran en sí mismos, sino que se esparcen y se entrelazan generando una serie de encuentros y desencuentros a nivel de las instituciones jurídicas, de las cuales cabe destacar aquí al concepto del propio derecho de gentes.

Esta forma de concebir la política tiene consecuencias evidentes en la gestación y comprensión del Derecho ${ }^{28}$, lo que nos dará como resultado un punto esencial a considerar: las instituciones jurídicas en nuestro continente no son puras, sino que reciben herencias que entre sí pueden ser contradictorias. Mientras que, desde una opción hispánica, con toda la dote neotomista, el Derecho es uno que apunta hacia el bienestar social, que permite una cohesión entre sus componentes; la opción inglesa es una que se inclina por la supervivencia y, por ello, en la individualidad y el sentido pragmático del Derecho: las instituciones jurídicas sirven para los fines egoístas de los individuos, para su perduración.

En el presente artículo, se ha buscado examinar la definición del derecho de gentes desde las visiones de Silva Santisteban y Bello, ambas en calidad de punto de partida y muestra para examinar un debate de quienes sostienen la juridicidad de este Derecho, frente a aquellos que lo niegan, considerando también, como marco general, la tensión existente entre la opción hispánica e inglesa en la configuración de estos dos pensamientos distintos. En ese sentido, en el debate entre Silva Santisteban y Bello, es necesario tomar consideración, por un lado, de la construcción

28 El adagio ubi societas, ibi ius (“donde hay sociedad, existe derecho") es fundamental aquí, dado que las características de una sociedad conducirán a la forja de las instituciones y nociones jurídicas, sin negar con ello, la precedencia de herencias históricas que moldean y que resultan ser puntos de partida en la elaboración de las normas o conceptos jurídicos. 
de Francisco de Vitoria, así como de su sucesor Grocio; y, por otro lado, la visión de Hobbes y Bentham, respectivamente.

En ese esquema, de la lectura atenta de los textos de Vitoria y Grocio, se puede identificar que para ellos existe un derecho de gentes universal que es capaz de exigir a todos sus componentes una debida obediencia por los valores que consagra. En cambio, la apreciación de las posturas de Hobbes y Bentham - pese a que este último fuera el que le diera nombre al derecho internacional (International law) (Rosales Zamora 2018: p. 17) - no les importa que tal derecho de gentes goce de universalidad: su enfoque es más pragmático, porque no ven el interés social como primero, sino que privilegian la posición de los Estados en su individualidad. En ese sentido, difícilmente conciben que los Estados acepten renunciar a su soberanía con facilidad por el bien común.

Debe recordarse que Silva Santisteban y Bello, siendo beneficiarios de todas estas herencias intelectuales, no son herederos pasivos, sino que son capaces de asimilar lo mejor de cada tradición y plantear relecturas desde una posición de consolidación de sus propios Estados, como lo llega a sostener Becker Lorca (2010: pp. 521-523). Recordemos, en ese sentido, que Silva Santisteban y Bello pertenecieron a Estados que habían surgido recientemente a la vida independiente y que buscaban sobrevivir a cualquier tipo de ofensiva de las potencias coloniales $^{29}$.

Luego de la revisión de las posturas de Silva Santisteban y Bello sobre la definición del derecho de gentes, se puede encontrar que el primero es un sucesor de Vitoria y el segundo, de Hobbes y Bentham. Y esto se percibe mejor apreciando que Silva Santisteban reconoce la juridicidad del derecho internacional, es decir, lo concibe como Derecho y, por lo tanto, admite su fuerza jurídica. En contraste, Bello, si bien desarrolla las principales instituciones del derecho internacional, su concepción del derecho de gentes es la de una recopilación de normas, pero no de un sistema jurídico en sí. Como lo afirma el mismo Silva Santisteban, al señalar como "[...] fin del derecho internacional la seguridad y bienestar común, o en lenguaje de Bentham, la utilidad general; y por una consecuencia lógica se deduce de sus doctrinas la negación del derecho internacional, supuesto que no lo concibe como colección de leyes, cuya existencia implica la necesidad de una autoridad superior, de que carecen las naciones" (1856: p. 42).

Este desencuentro de posturas parte de que Silva Santisteban admite la sociabilidad de las naciones en el marco de la justicia, mientras que Bello, como lo demuestra su propia biografía, fue formado en Londres y bebió de los escritos de Bentham, alejándose de los ideales hispánicos descritos por Morse. En ese sentido, la reconciliación entre ambos autores es difícil de admitir, porque son hijos de su tiempo, pero de fuentes diferentes: Silva Santisteban es un iusnaturalista que pretende construir un derecho de gentes que parta de su originalidad hispánica, ese ideal de justicia vitoriano, mientras que Bello adopta el enfoque pragmático que le precede: una visión que, en el fondo, considera más la guerra que la paz como elemento trascendental configurador de las relaciones entre las naciones y, por lo tanto, se vuelve un conspicuo perseguidor de la utilidad general.

29 Aquí cabe resaltar que, para el año de 1824, el presidente Monroe sostendrá que “América pertenece a los americanos", con el propósito de evitar la vuelta a la dominación de las potencias europeas hacia estas tierras. (Cassese 2013: p. 39). 
Se debe aquí advertir que Silva Santisteban, con mucha perspicacia, fue capaz de identificar a Bello como enemigo ideológico en su obra. Es más el autor peruano afirma que "es tan seductor el lenguaje de bien público, halaga y fascina tanto que no solo ha sido el talismán de las naciones, sino que los publicistas de nuestros días aceptan y propagan sus doctrinas [...] Bello lo designa con el nombre de seguridad y bienestar común. Las palabras son varias, la idea una misma: interés, bienestar, felicidad, seguridad, todo indica la utilidad y nada más; todos son discípulos de Bentham" (Silva Santisteban 1856: p. 50).

Silva Santisteban sostiene la posibilidad de la justicia y llama a Bello de negador del derecho internacional, al ser discípulo de Bentham, y, en el fondo, un defensor de la utilidad. En el fondo, los valores que se contraponen entre sí, de estos autores, son la justicia y la utilidad, la abstracción y la individualización. Tales ideales generan perspectivas y resultados diferentes en el esquema de la relación entre las naciones. La adopción de la justicia como valor demuestra que Silva Santisteban es un iusnaturalista, mientras que en Bello vemos un utilitarista. El primero sostendrá el carácter científico del Derecho y el segundo se limitará, pese a su nivel de instrucción, el cual es innegable, a cumplir "el papel de compilador al de reformador" (1856: p. 42).

\section{Conclusiones}

El estudio histórico del derecho internacional realizado a través de la figura de Silva Santisteban y Bello demuestra que estos autores, siendo pensadores del ius gentium pertenecientes a las Américas, son una prueba de que la aproximación hacia el derecho de gentes en el siglo XIX no puede seguir haciéndose desde una tesis tradicionalista, por la cual se asuma que la matriz europea se haya trasladado sin más a nuestro continente, sino que es fundamental partir de una mirada crítica: Silva Santisteban y Bello son muestras, cada uno con su propio perfil, de no ser simplemente receptáculos del derecho internacional construido en Europa.

Silva Santisteban y Bello encarnan el debate que Morse retrata en $O$ espelho de Próspero: cultura e idéias nas Américas entre la opción ibérica y la opción inglesa, entre el neotomismo y el empirismo, respectivamente. En el fondo, es un debate que se produce entre los que afirman y niegan el derecho internacional y su carácter científico. Silva Santisteban sería un descendiente de Vitoria y Bello un descendiente de Hobbes y Bentham. En ese plano, Silva Santisteban parte, entre otros motivos, de criticar la obra Principios de derecho internacional de Bello por haber renunciado a la búsqueda de cientificidad del derecho de gentes. Además, Silva Santisteban parece ser mucho más consciente que Bello sobre el perfil histórico de su posición y lo critica ferozmente, calificándolo de empirista.

Como se ha explorado en este trabajo, Silva Santisteban constituye un formulador del derecho internacional en su carácter de ciencia, sin dejar por ello de considerarla basada en los "principios eternos de justicia". En contraste, Bello únicamente es capaz, y en ello coincide con Silva Santisteban, de sostener que el derecho internacional es una lista o recopilación de normas, sin preocuparse por dotar a esta rama de un carácter científico. En ese sentido, las definiciones del derecho internacional que manejan estos autores demuestran no solo que uno es un afirmador del derecho internacional y el otro un negador de su carácter científico, sino que, además, permite visualizar la lucidez de Morse de graficar el debate entre neotomismo y empirismo en la 
formación de la historia de las Américas, porque esta desciende en las personas de estos autores y se produce en un área tan específica como la del derecho de gentes.

\section{Bibliografía}

Anghie, A. (1996). Francisco de Vitoria and the Colonial Origins of International Law. En: Social \& Legal Studies, vol. 5, n. ${ }^{\circ}$ 3, pp. 321-336.

Armitage, D.; Pitts, J. (2017). The Law of Nations in Global History, Oxford: Oxford University Press.

Aristóteles (1989). Politeia (La Política), Bogotá: Instituto Caro y Cuervo (Ed. por Briceño Jauregui).

Basadre Ayulo, J. (2001). Los juristas de la República del Perú en el siglo XIX: Francisco García Calderón Landa. En: Revista de Estudios Histórico-Jurídicos, vol. XXIII, pp. 471-486.

Becker Lorca, A. (2010). Universal International Law: Nineteenth-Century Histories of Imposition and Appropriation. En: Harvard International Law Journal, vol. 51, n. ${ }^{\circ}$ 2, pp. $475-$ 552.

Bello, A (1981). Obras completas de Andrés Bello, vol. X (Derecho Internacional), Caracas: Fundación La Casa de Bello.

Bello, A. (1873). Principios de derecho internacional, 3ra. Ed., Chile: Pablo Dupont.

Brett, A. Chapter 46, Francisco de Vitoria (1483 - 1546) and Francisco Suárez (1548 - 1617). En: Fassbender, B. y Peters, A. (2012). The Oxford Handbook of the History of International Law, Oxford: Oxford University Press.

Cassese, A. (2013). Diritto internazionale (a cura di Paola Gaeta), Bolonia: Il Mulino.

Clapham, A. (2012). Brierly's Law of Nations, Oxford: Oxford University Press.

Daillier, P. ; Forteau, M.; Pellet, A. (2009). Droit international public, Paris: L.G.D.J.

De Vitoria, F. (1975) Relecciones de indios y el derecho de guerra, Madrid: Espasa Calpe, Tercera edición.

De Vitoria, F. (2008). Relectio de potestate civili. Estudios sobre su filosofía política. (Ed. Cordero Pando, J.), Madrid: Consejo Superior de Investigaciones Científicas.

Flórez, G. (1999). De la sociedad feudal a la génesis del Estado moderno en Europa Occidental. En: Agenda Internacional, vol. 6, n. ${ }^{\circ}$ 12, pp. 113-122.

Franca, M. (2006). Historia y Razón del Paradigma Westfaliano. En: Revista de Estudios Políticos (nueva época), n. ${ }^{\circ} 131$, pp. 87-111. 
Gómez Robledo, A. (1989). Fundadores del derecho internacional, México: Universidad Nacional Autónoma de México.

Hobbes, T. (1987). De Cive, Oxford: Oxford University Press (Ed. por Warrender).

Hobbes, T. (1998). Leviathan, Oxford: Oxford University Press (Ed. por Gaskin).

Iannarone, R. (1970). La maturazione delle idee coloniali in Francisco de Vitoria. En: Angelicum, Vol. 47, N. ${ }^{\circ}$, pp. 3-43.

José Contreras, F. (2016). La filosofía del Derecho en la Historia. Madrid: Tecnos (2 Ed.).

Miaja de la Muela, A. (1965). El Derecho "Totius Orbis" en el pensamiento de Francisco de Vitoria. En: Revista Española de Derecho Internacional, vol. 18, n. ${ }^{\circ}$ 3, pp. 341-364.

Morse, R. (1988). O espelho de Próspero, cultura e idéias nas Américas, trad. Neves, P., Sao Paulo: Companhia das Letras.

Murphy, Sean. (2012). Principles of International Law, Estados Unidos de América: Thomson Reuters.

Neff, S (2014). Justice Among Nations. A History of International Law, Estados Unidos de América: Harvard University Press.

Olano, H. (2007). Andrés Bello, el jurista de las Américas. En: Dikaion, año 21, n. ${ }^{\circ}$ 16, pp. 6773.

Orakhelashvili, A. (2011). The relevance of theory and history - the essence and origins of international law. En: Orakhelashvili, A. (Ed.) Research Handbook on the Theory and History of International Law, Cheltenham: Elgar.

Osterhammel, Jürgen. (2015). La transformación del mundo. Una historia del siglo XIX, Barcelona: Planeta.

Otero Parga, M. (2017). ¿Influyó Suárez en el pensamiento de Andrés Bello? En: Anales de la Cátedra Francisco Suárez, n. ${ }^{\circ}$ 51, pp. 79-100.

Reale, G. ; Antiseri, D. (1988). Historia del pensamiento filosófico y científico. Del romanticismo hasta hoy, vol. 3, Barcelona: Herder.

Rosales Zamora, P. (2018). Sobre la vocación por el Derecho internacional público: consideraciones en torno a su estudio. En: Ius Inter Gentes, Revista de Derecho Internacional y Relaciones Internacionales, año $1, \mathrm{n} .^{\circ} 1$, pp. 15-30. 
Sánchez de Puerta Trujillo, F. (2006). Los tipos ideales en la práctica: significados, construcciones, aplicaciones. En: Revista de Metodología en Ciencias Sociales, N. ${ }^{\circ} 11$, pp. 1132.

Silva Santisteban, J. (2018). Curso de derecho internacional o de gentes (1856), Lima: Centro de Estudios Constitucionales.

Truyol y Serra, A. (1998). Historia del Derecho Internacional Público. Madrid: Tecnos.

Walz, G. (1943). Derecho internacional y crítica de sus negadores. Madrid: Revista de Derecho Privado.

Weber, M (2016). La ética protestante y el «espíritu» del capitalismo, Madrid: Alianza editorial (Ed. Por Abellán, J.). 